\title{
HIGH PREVALENCE OF CERVICAL SQUAMOUS INTRAEPITHELIAL LESIONS IN WOMEN ON ANTIRETROVIRAL THERAPY IN CAMEROON: IS TARGETED SCREENING FEASIBLE?
}

\author{
Julius Atashili ${ }^{1,2,3}$, Adaora A Adimora ${ }^{3}$, Peter M Ndumbe ${ }^{1,2}$, George M Ikomey ${ }^{1,2}$, Allen C \\ Rinas $^{3}$, Evan Myers ${ }^{3}$, Joseph Eron ${ }^{3}$, Jennifer S Smith ${ }^{3}$, and William C Miller \\ Julius Atashili: atashili@yahoo.ie; Adaora A Adimora: adimora@med.unc.edu; Peter M Ndumbe: pndumbe@yahoo.com; \\ George M Ikomey: gikomey@yahoo.com; Allen C Rinas: arinas@med.unc.edu; Evan Myers: evan.myers@duke.edu; \\ Joseph Eron: joseph_eron@unc.edu; Jennifer S Smith: jsssmith@email.unc.edu; William C Miller: bill_miller@unc.edu \\ ${ }^{1}$ University of Buea, Cameroon \\ 2University of Yaounde I, Cameroon \\ ${ }^{3}$ University of North Carolina at Chapel Hill, North Carolina, USA
}

\section{Abstract}

Background-Cervical cancer is the most common cancer in women in low-income countries. Although cervical cancer incidence and mortality is higher in HIV-positive women, resource limitations restrict the implementation of systematic screening programs in these women. We explored the potential for targeted screening by assessing the prevalence, severity and predictors of cervical squamous intra-epithelial lesions (SILs) in HIV-positive women in Cameroon.

Methods and findings-We conducted a cross-sectional study of women on antiretroviral therapy in Cameroon. Socio-demographic, behavioral, and clinical information was obtained from eligible women. Cervical exfoliated cells were then collected, a conventional cytology performed and epithelial lesions classified according to the Bethesda 2001 system.

A total of 282 women, aged 19 to 68 years, were enrolled in this study. The median CD4 count was 179 cells/microliter (interquartile range: 100 to 271). SILs were detected in $43.5 \%$ of the 276 women with satisfactory samples: including atypical squamous cells of unknown significance (ASCUS) 0.7\%, low-grade SIL (LSIL) 25.0\%, atypical squamous cells, cannot exclude high grade

(C) 2011 Published by Elsevier Ltd.

Author contributions

Study conception and design: JA, JSS, AAA, JE, WCM, EM

Study implementation: JA, JSS, PMN, GMI, ACR

Analysis and review: JA, GMI, ACR, JSS, WCM

Manuscript writing and revisions: JA, PMN, GMI, JSS, AAA, JE, WCM, EM

CONFLICTS OF INTEREST

JA has received honoraria from GSK and research supplies from Digene. JSS has received research grants, honoraria, or consulting fees during the last three years from GSK, Digene and GenProbe. EM has received research funding and done consulting for Merck \& Co.

Ethical review

Study approved by the Cameroon National Ethics Committee and the University of North Carolina at Chapel Hill's Biomedical IRB.

Publisher's Disclaimer: This is a PDF file of an unedited manuscript that has been accepted for publication. As a service to our customers we are providing this early version of the manuscript. The manuscript will undergo copyediting, typesetting, and review of the resulting proof before it is published in its final citable form. Please note that during the production process errors may be discovered which could affect the content, and all legal disclaimers that apply to the journal pertain. 
lesions (ASC-H) 14.5\%, and high-grade SIL (HSIL) 3.3\%. None of the demographic or clinical characteristics considered significantly predicted the presence of any SILs or the presence of severe lesions requiring colposcopy.

Conclusion-The prevalence of SIL in women on antiretroviral therapy in Cameroon was high underscoring the need for screening and care in this population. In the absence of any accurate demographic or clinical predictor of SIL, targeted screening does not seem feasible. Alternative affordable screening options need to be explored.

\section{Keywords}

Cervical neoplasm; HIV; Screening; Cameroon; Epidemiology

\section{INTRODUCTION}

Cervical cancer is the most common cancer in women in low-income countries[1]. Compared to immuno-competent women, HIV-positive women have a higher prevalence, incidence and progression rate of precancerous cervical lesions[2,3]. Enhancing early detection and treatment of precancerous lesions through screening could reduce the burden of cervical cancer in these HIV-positive women[4].

Despite the relatively high risk of precancerous and cancerous cervical lesions in HIVpositive women, unlike many other opportunistic infections, the current management of women initiating antiretroviral therapy (HAART) in most low-income countries does not include a systematic screen for cervical cancer or precancerous lesions. Targeted screening among HIV-positive women could potentially increase the cost-effectiveness of screening in these resource-limited settings by increasing the ratio of the number of cases detected per screening test. However, for targeted screening to be effective socio-demographic and clinical factors associated with a higher prevalence and severity of lesions need to be identified.

In this paper, we describe the prevalence and severity of cervical epithelial lesions in women initiating HAART in Cameroon and assess the clinical predictors of lesions in these women. Clinical risk scores are also developed based on the aforementioned predictors and their potential performance assessed.

\section{PATIENTS AND METHODS}

\section{Ethics statement}

This study was approved by the Cameroon National Ethics Committee and the University of North Carolina at Chapel Hill's Biomedical IRB. All participants provided written informed consent prior to inclusion in the study.

\section{Study design and study population}

We conducted a cross-sectional study of HIV-positive women recruited from three HIV-care clinics in Cameroon: the Bamenda Provincial Hospital AIDS Treatment Center (ATC), the Limbe Provincial Hospital ATC and the Nylon District Hospital ATC in Douala. The clinics are all located in urban areas in Cameroon but provide regular care to patients from surrounding urban and peripheral rural areas. Consecutive HIV-positive women receiving care in these clinics, between August and September 2008, were invited to participate in the study. Women aged 18 years or more, who initiated HAART within a year preceding study enrollment and consented to study procedures, were eligible. Women who were either pregnant, having menses or had a previous total hysterectomy were excluded. 


\section{Study procedures}

Three research assistants with previous medical training were trained on study procedures and cervical sampling to optimize the quality of cervical smears. After providing usual care, clinicians at each site referred the patients to our research assistants who then explained the study to each eligible patient. After obtaining written informed consent and verifying eligibility, data on demographic and clinical characteristics was collected with the aid of a standardized questionnaire. The assistants then collected cervical smear samples using conventional methods. Two slides were made for each participant. The slides were immediately fixed using a $95 \%$ ethanol solution and stored at room temperature for the duration of patient enrollment. These slides were sent for analysis and storage in the laboratory of the Center for the Study and Control of Communicable Disease (CSCCD) of the Faculty of Medicine and Biomedical Sciences, Yaoundé, Cameroon.

Slides were stained by the Papanicolau's method and examined under the microscope by a trained cytologist. Each slide was scored according to the Bethesda 2001 system as unsatisfactory, negative, atypical squamous cells of uncertain significance (ASCUS), lowgrade squamous intraepithelial lesions (LSIL), atypical squamous cells, cannot exclude high grade lesions (ASC-H), high-grade SIL (HSIL), or invasive cervical cancer[5]. Cytology readings were conducted blinded of clinical characteristics. For quality control purposes, both research assistants and cytologists received specific training related to the study, two slides were made and analyzed for each patient (the most severe result was considered the final result, in case of differences between both slides), and slides with lesions were doublechecked by a cytologist external to the study (differences were resolved by consensus). Furthermore, a subset of 25 slides were reviewed by an experienced cytologist at the University of North Carolina at Chapel Hill - the percentage agreement on the presence of lesions or not was $76 \%$, (kappa $=0.49)$ while the percentage agreement on lesions being ASC-H/HSIL or not was $60 \%(\mathrm{kappa}=0.26)$. The potential impact of these limitations with conventional cytology was assessed in sensitivity (bias) analyses[6].

\section{Statistical analysis}

Data collected were entered into MS Access interface on Epi-info 2000. Statistical analyses were conducted using SAS version 9.2 (SAS institute inc, Cary NC) and STATA version 10 (STATA corps, Texas USA). Two outcomes, based on cervical cytology, were considered: 1) Prevalent cervical lesions (defined as the presence of any cervical epithelial lesions); 2) Prevalent ASC-H/HSIL (lesions requiring colposcopy). Participant's age, marital status, parity, number of lifetime sex partners, age at first sexual intercourse, history of hormonal contraception, history of exposure to cigarette smoke, CD4 count, and AIDS clinical stage were considered as potential clinical predictors of lesions. These characteristics were chosen based on the ease with which they can be elicited and recorded in a clinical setting and previous literature describing their association with the presence of cervical lesions or a plausible etiological role.

All predictors considered were included in multivariable analyses. For each outcome, an initial (full) model that includes all the predictors was created. We then attempted to determine a reduced model based on a parsimonious subset of clinically and statistically significant predictors identified by a stepwise backward elimination strategy[7]. A final reduced model was thus obtained retaining only predictors that when dropped resulted in a likelihood ratio test p-value $<0.2$ or a more than $10 \%$ change in the area under the curve of the ROC plot (c-statistic). A reduced model was possible only for the prediction of prevalent SIL (and not for the prediction of ASC-H/HSIL). 
The models developed were based on the full sample. However the internal validity of the models' performance was ascertained by implementing the models to three subsets of the sample corresponding to each study site. The c-statistics for the models were all within $20 \%$ of the model implemented on the full sample.

One objective of this analysis was to identify if any clinical predictor(s) could be used in a resource-limited settings to develop a targeted screening approach. We thus developed and assessed potential risk scores for targeting screening only to patients more likely to have lesions. Risk scores were developed from each of the three final models: the two models for predicting the presence of any lesion (the full model and the reduced model) and the full model for predicting the presence of ASC-H/HSIL. The numeric score assigned to each predictor was based on the model slope coefficients. To allow for a simple and feasible application in clinical settings, each predictor score was obtained by multiplying the model slope coefficients by 10 and then rounding to the lower integer. The aggregate risk score was based on the sum total of each predictor score. We assessed the performance (sensitivity, specificity, positive and negative predictive values) of each risk score for targeting $25 \%, 50 \%$ and $75 \%$ of women. We also evaluated the total errors that would result from implementing either targeted screening based on the risk scores versus universal or no screening. Total unweighted errors were estimated as the sum of 'false negative' and 'false positive' errors, respectively defined as the number of patients with no lesion being screened and the number of patients with lesions not being screened. Total weighted errors were also estimated taking into account the relative cost (both monetary and non-monetary) associated with having a 'false negative' versus a 'false positive' error.

\section{RESULTS}

\section{Study population}

Altogether 282 women were enrolled in this study. Participants' age ranged from 19 to 68 years, with a mean of 36 years. Most participants (73.4\%) were from urban areas (Table 1). As many as $26.9 \%$ were widowed, while $21.3 \%$ had never been married. The median parity was 2 (range $0-11$ ). Active tobacco exposure (2.5\%) and oral contraceptive pill usage (23.8\%) was relatively infrequent. The number of lifetime partners exceeded 5 in $25.2 \%$ of participants. A history of genital warts was reported by $7.1 \%$ of participants while $43.3 \%$ could not say if they previously had genital warts or not.

HIV diagnosis had preceded study enrolment by 18.5 months on average (range 0-136 months). The median CD4 count was 179 cells/microliter (interquartile range: 100 to 271). The vast majority of patients $(80.9 \%$ ) had advanced HIV disease (WHO HIV clinical stages III/IV). Only $2.1 \%$ were certain they had previously had a Pap smear.

\section{Prevalence and severity of lesions}

The prevalence of SIL was $43.5 \%$ (95\%CI: $37.5,49.6 \%$ ) in the 276 women with satisfactory samples. The prevalence of specific abnormalities was ASCUS 0.7\% (95\%CI: 0.09, 2.3\%), LSIL 25.0\% (95\%CI: 20.0, 30.5\%), ASC-H 14.5\% (95\%CI: 10.6, 19.2\%), and HSIL 3.3\% (95\% CI: $1.5,6.1 \%$ ). The overall prevalence of ASC-H/HSIL was $17.8 \%$ (95\% CI: 13.4 , $22.8 \%)$.

\section{Association of clinical predictors and prevalent SIL}

Most of the clinical factors assessed were either weakly associated with prevalent SIL or had a poor precision and were statistically insignificant (Table 2). Compared to women from urban areas, SIL tended to be more frequent in women from rural areas (adjusted OR: 1.68; 95\% CI: $0.88,3.18$ ). SIL tended to be more frequent in women aged 26-59 compared to all 
other women (aOR: $1.57 ; 0.65,3.81)$. Although estimates were relatively imprecise, the odds of SIL tended to be lower in women with HIV stage III/IV compared to women in stage I/II (aOR: 0.63; 95\% CI: 032, 1.23). The odds of SIL did not differ substantially by marital status, educational status, previous exposure to tobacco smoke, previous pill usage, parity, age at first sex, time since HIV diagnosis or CD4 count (all aORs were more than 0.8 and less than 1.25).

The overall predictive value of the model with all these variables was relatively low with a c-statistic of 0.60 . A reduced model included only two predictors: participants' residence (rural vs. urban aOR 1.58 (95\% CI: 0.87, 2.87) and HIV clinical stage (HIV stage III/IV vs. I/II aOR 0.61 (95\% CI: $0.31,1.19))$, with a c-statistic of 0.58 .

\section{Association of clinical predictors and lesions requiring colposcopy/biopsy}

Bivariable and multivariable associations with ASC-H/HSIL were relatively weak (Table 2). Although not statistically significant, ASC-H/HSIL tended to be more frequent in women who reported age at first sexual intercourse less than 16 years (aOR: 1.27 ; 95\% CI: 0.64 , 2.53 ) as well as in patients with CD4 counts below 200 (aOR: 1.49; 95\%CI: 0.72, 3.07). ASC-H/HSIL tended to be less frequent in women self-reporting exposure to tobacco smoke (aOR: $0.66,95 \%$ CI: $0.33,1.34$ ). The odds of ASC-H/HSIL did not differ substantially by age, marital status, education, residence, age, previous pill usage, parity, HIV clinical stage, or time since HIV diagnosis (all aORs were more than 0.8 and less than 1.25). With a cstatistic of 0.59 , the overall predictive value of the model with all these variables was also low.

\section{Assessment of clinical risk scores for targeted screening}

The clinical risk scores developed are defined and assessed in Tables 3 and 4. Overall the classification accuracy based on these scores were all less than $70 \%$. If the risk score based on the full model for any SIL was used to target the screening of $25 \%$ of women, then only $35.7 \%$ of women with lesions would have been screened while $23 \%$ of women with no lesions would be screened as well. If the target was to screen $25 \%$ of women with ASC-H/ HSIL, then using the score based on the full model predicting ASC-H/HSIL would result in $38.1 \%$ of women with ASC-H/HSIL lesions being screened while $26.7 \%$ of women with no ASC-H/HSIL would also be screened.

It is worth noting that despite the relatively poor performance of these scores, their value compared to universal screening or no screening was a function of the relative weight given to 'false negative' errors compared to 'false positive' errors. In screening for any lesion, when 'false negative' errors were considered equal to 'false positive' errors, then the total error rates associated with targeting 50\% (406 errors per 1000 women) or 25\% (410 errors per 1000 women) of women was lower than each of universal screening (565 errors per 1000 women) or no screening (435 errors per 1000 women). However, as the relative cost of 'false negative' errors (compared to 'false positive' errors) increased, universal screening tended towards having the least total errors while the total errors associated with screening fewer proportions of patients increased (Figure 1). Similar trends were observed in assessing potential screening for ASC-H/HSIL only (Figure 2). While no screening was associated with the least errors when 'false negative' errors were considered equal to 'false positive' errors, increasing the proportion of women screened was a preferable option when the former errors were at least five times the latter.

\section{Bias analyses}

We conducted bias analyses assessing what the true population prevalence of lesions could be considering the inaccuracies in conventional cytology. Our analysis showed that a lower 
sensitivity of cytology would mean that the study tended to underestimate the true prevalence while a lower specificity would have resulted in the study overestimating the true prevalence. Our data were compatible with a true population prevalence of lesions ranging from $19.3 \%$ (when the sensitivity was $100 \%$ and the specificity $70 \%$ ) to $87.0 \%$ (when the sensitivity was $50 \%$ and specificity $100 \%$ ). Meanwhile the prevalence of ASC-H/HSIL could be as low as $0 \%$ (if the specificity was $80 \%$ or less) and as high as $35.6 \%$ (when sensitivity was $50 \%$ and specificity $100 \%$ ).

\section{DISCUSSION}

To appraise the need and potential for targeted screening for cervical cancer in HIV-positive women in resource-limited settings, we assessed the prevalence, severity and predictors of SIL in women initiating antiretroviral therapy in Cameroon. We document that the prevalence of precancerous lesions is high in these women. Approximately twenty percent of these women have lesions severe enough to warrant colposcopy. In this study population, readily available demographic and clinical factors, both individually and as a group, did not accurately distinguish women with lesions from those without.

While the overall prevalence of any cervical lesion in this population appears high (43\%), it is within the range described in women in similar conditions. Among HIV-positive pregnant women in Yaounde, Cameroon, lesions were detected in 40\%, including 12\% with high grade lesions[8]. The prevalence of SIL in HIV-seropositive women living in sub-Saharan Africa has ranged from 15 to $48 \%$ in Burkina Faso[9], Kenya[10], South Africa[11,12], Zimbabwe[13], Rwanda[14], Malawi[15] and Cote d'Ivoire[16]. The highest prevalence of SIL $(76 \%)$ so far was detected in a study of 150 HIV-positive women in Zambia who had a median CD4 count of 165/uL[17]. Although an unusually low prevalence of $7 \%$ was reported in a study of 691 women in Tanzania, the vast majority (86\%) of these women were in WHO clinical stage I and only $12 \%$ had CD4 count less than 200[18].

The prevalence in our study could be influenced by study population characteristics or the accuracy of cytology results. Though we sampled in three different clinics, the sample may not have been exactly representative of all HIV-positive women in Cameroon as the clinics were conveniently selected and not randomly chosen. We do not however expect the difference to be substantial as the clinics sampled offer care to a very high number of patients in regions with the highest HIV-prevalences and our participant characteristics were similar to those reported nationwide[19]. Potential errors due to the Pap smear's low sensitivity could actually mean that our prevalence is an underestimate. Nevertheless, the sensitivity of cytology in this study was assured by research assistants being trained to insure adequate sample collection, collecting two slides per patient to reduce sampling error and having the interpretations validated by an experienced cytologist. The latter review suggested a tendency of the initial cytologist to over classify participants as having lesions. Our bias analyses however shows that the lowest true population prevalence of lesions compatible with our data was in the order of $19 \%$ which is still relatively high.

The high prevalence of cervical lesions in this population reiterates the current need to offer screening and care for cervical precancerous lesions in HIV-positive women. Despite being at increased risk for cervical cancer, less than 5\% of participants in our study had previously been screened. Access to antiretrovirals has dramatically increased in developing countries. While the increased survival that is expected to accompany this increased access the effect on cervical precancerous lesions is expected to be moderate at best, as HAART has a very limited effect, if any, on HPV persistence and the progression of lesions[20]. Even while on antiretrovirals women will still need to be screened or receive other preventive care for cervical cancer. 
We are not aware of any other study that assessed the potential for clinical risk scores for targeted cervical cancer screening in HIV-positive women. Because most studies were conducted to establish the association between HIV infection and the presence of SIL, very few assessed the risk factors specific to HIV-positive women. Amongst the few that did, immunosuppression (low CD4 count), high HIV viral load and or infection with high-risk HPV types tended to be associated with the presence of SIL[10, 14, 16, 17]. While our sample size was adequate for estimating prevalence, only a limited number of covariates could be considered as potential predictors. Predictors were thus judiciously chosen taking into account the established literature and the ease of clinical assessment. Women with lower CD4 counts and younger age at first sex thus appeared more likely to have severe lesions. We did not consider HIV viral load as resource limitations render it difficult to ascertain in most patients, making it of little clinical use in this setting. Unexpectedly, women who self-reported exposure to cigarette smoke or a history of taking contraceptive pills tended to be less likely to have lesions. We attribute these apparent lower odds to these factors being indicators of higher socioeconomic status in these settings. It is not clear why women with more advanced clinical HIV disease appeared less likely to have lesions. Nevertheless this association was relatively imprecise.

Considered as a group, the clinical predictors only slightly performed better than chance in differentiating women who had lesions (or women with severe lesions) from those without. For comparison the commonly used Framingham cardiovascular risk score had a c-statistic in the order of $0.65-0.70$ in a population of diabetics in the UK[21]. Nonetheless, the choice between universal screening, targeted screening or no screening would depend on how much policy makers value the cost of 'false negative' errors relative to the cost of 'false positive' errors. We expect that most would attribute a higher long-term cost to 'false negative' errors than to 'false positive' errors as cancer can develop in the former, while the latter would only result in an unnecessary pap smear. With this being the case, strategies advocating for more screening (thus with higher sensitivity and lower 'false negatives') will tend to be better options. Formal cost-effectiveness analyses may aid in further clarifying which option is best in each setting.

In addition to prevalence, the need to screen would also depend on the progression of lesions towards self-resolution or cancer. It was not possible to assess the progression or regression of lesions, given the cross-sectional nature of our study. Further studies would need to assess the long-term progression of lesions in HIV-positive women in this setting.

In conclusion, the prevalence of SIL in women initiating antiretroviral therapy in Cameroon was high. This high prevalence, in light of the potential to treat precancerous lesions when detected early and the limited role of HAART on the progression of lesions, underscores the need for screening in this population. Targeted screening for SIL using clinical and demographic predictors among HIV positive women did not appear feasible. In the absence of any accurate demographic or clinical predictor of SIL, alternative affordable screening options need to be explored. A prospective study of the long-term evolution of these lesions and their determinants, involving a sufficiently large sample of women, is needed to further guide policy decisions.

\section{Acknowledgments}

\section{FUNDING}

This work was supported by a Fogarty fellowship provided by the NIH Fogarty AIDS International Training and Research Program to the University of North Carolina at Chapel Hill [DHHS/NIH/FIC 5 D43 TW01039-08] and Developmental Award from the University of North Carolina at Chapel Hill's, Center for AIDS Research [NIH \#9P30 AI 50410]. Dr. Adimora's contribution was partially supported by NIH 1K24HD059358-01. 
George Ikomey, the cytologist on the study was trained with support from an UICC (International Union against Cancer) International Cancer Technology Transfer (ICRETT) Fellowship.

The funders had no role in study design, data collection and analysis, decision to publish, or preparation of the manuscript.

\section{References}

1. WHO. Preparing for the introduction of HPV vaccines: policy and programme guidance for countries. WHO; Geneva: 2006.

2. Palefsky J. HPV infection and HPV-associated neoplasia in immunocompromised women. Int $\mathbf{J}$ Gynaecol Obstet. 2006; 94 (Suppl 1):S56-64.

3. De Vuyst H, Lillo F, Broutet N, Smith JS. HIV, human papillomavirus, and cervical neoplasia and cancer in the era of highly active antiretroviral therapy. Eur J Cancer Prev. 2008; 17(6):545-54. [PubMed: 18941376]

4. Franceschi S, Jaffe H. Cervical cancer screening of women living with HIV infection: a must in the era of antiretroviral therapy. Clin Infect Dis. 2007; 45(4):510-3. [PubMed: 17638204]

5. Solomon D, Davey D, Kurman R, Moriarty A, O'Connor D, Prey M, et al. The 2001 Bethesda System: terminology for reporting results of cervical cytology. JAMA. 2002; 287(16):2114-9. [PubMed: 11966386]

6. Rothman, KJ.; Greenland, S.; Lash, TL. Modern Epidemiology. 3. Philadelphia: Lippincott Williams\& Wilkins; 2008.

7. Harrell, FE. Regression Modeling Strategies: With Applications to Linear Models, Logistic Regression, and Survival Analysis. New York: Springer; 2001.

8. Mbu ER, Kongnyuy EJ, Mbopi-Keou F, Tonye RN, Nana PN, Leke RJ. Gynaecological morbidity among HIV positive pregnant women in Cameroon. Reprod Health. 2008; 5:3. [PubMed: 18598359]

9. Didelot-Rousseau MN, Nagot N, Costes-Martineau V, Vallès X, Ouedraogo A, Konate I, et al. Human papillomavirus genotype distribution and cervical squamous intraepithelial lesions among high-risk women with and without HIV-1 infection in Burkina Faso. Br J Cancer. 2006; 95(3):35562. [PubMed: 16832413]

10. Yamada R, Sasagawa T, Kirumbi LW, Kingoro A, Karanja DK, Kiptoo M, et al. Human papillomavirus infection and cervical abnormalities in Nairobi, Kenya, an area with a high prevalence of human immunodeficiency virus infection. J Med Virol. 2008; 80(5):847-55. [PubMed: 18360898]

11. Moodley M, Garib R. The significance of human papillomavirus infection detected by cervical cytology among women infected with the human immunodeficiency virus. J Obstet Gynaecol. 2004; 24(8):903-6. [PubMed: 16147648]

12. Gaym A, Mashego M, Kharsany AB, Walldorf J, Frohlich J, Karim QA. High prevalence of abnormal Pap smears among young women co-infected with HIV in rural South Africa implications for cervical cancer screening policies in high HIV prevalence populations. S Afr Med J. 2007; 97(2):120-3. [PubMed: 17404673]

13. Chirenje ZM, Loeb L, Mwale M, Nyamapfeni P, Kamba M, Padian N. Association of cervical SIL and HIV-1 infection among Zimbabwean women in an HIV/STI prevention study. Int J STD AIDS. 2002; 13(11):765-8. [PubMed: 12437897]

14. Leroy V, Ladner J, De Clercq A, Meheus A, Nyiraziraje M, Karita E, et al. Cervical dysplasia and HIV type 1 infection in African pregnant women: a cross sectional study, Kigali, Rwanda. The Pregnancy and HIV Study Group (EGE). Sex Transm Infect. 1999; 75(2):103-6. [PubMed: 10448362]

15. Motti PG, Dallabetta GA, Daniel RW, Canner JK, Chiphangwi JD, Liomba GN, et al. Cervical abnormalities, human papillomavirus, and human immunodeficiency virus infections in women in Malawi. J Infect Dis. 1996; 173(3):714-7. [PubMed: 8627037]

16. La Ruche G, Ramon R, Mensah-Ado I, Bergeron C, iomande’' M, Sylla-Koko F, et al. Squamous intraepithelial lesions of the cervix, invasive cervical carcinoma, and immunosuppression induced 
by human immunodeficiency virus in Africa. Dyscer-CI Group. Cancer. 1998; 82(12):2401-8. [PubMed: 9635533]

17. Parham GP, Sahasrabuddhe VV, Mwanahamuntu MH, Shepherd BE, Hicks ML, Stringer EM, et al. Prevalence and predictors of squamous intraepithelial lesions of the cervix in HIV-infected women in Lusaka, Zambia. Gynecol Oncol. 2006; 103(3):1017-22. [PubMed: 16875716]

18. Kapiga SH, Msamanga GI, Spiegelman D, Mwakyoma H, Fawzi WW, Hunter DJ. Risk factors for cervical squamous intraepithelial lesions among HIV-1 seropositive women in Dar es Salaam, Tanzania. Int J Gynaecol Obstet. 1999; 67(2):87-94. [PubMed: 10636052]

19. Mosoko JJ, Macauley IB, Zoungkanyi AC, Bella A, Koulla-Shiro S. Human immunodeficiency virus infection and associated factors among specific population subgroups in Cameroon. AIDS Behav. 2009; 13(2):277-87. [PubMed: 17694430]

20. Heard I, Palefsky JM, Kazatchkine MD. The impact of HIV antiviral therapy on human papillomavirus (HPV) infections and HPV-related diseases. Antivir Ther. 2004; 9(1):13-22. [PubMed: 15040532]

21. Guzder RN, Gatling W, Mullee MA, Mehta RL, Byrne CD. Prognostic value of the Framingham cardiovascular risk equation and the UKPDS risk engine for coronary heart disease in newly diagnosed Type 2 diabetes: results from a United Kingdom study. Diabet Med. 2005; 22(5):55462. [PubMed: 15842509] 


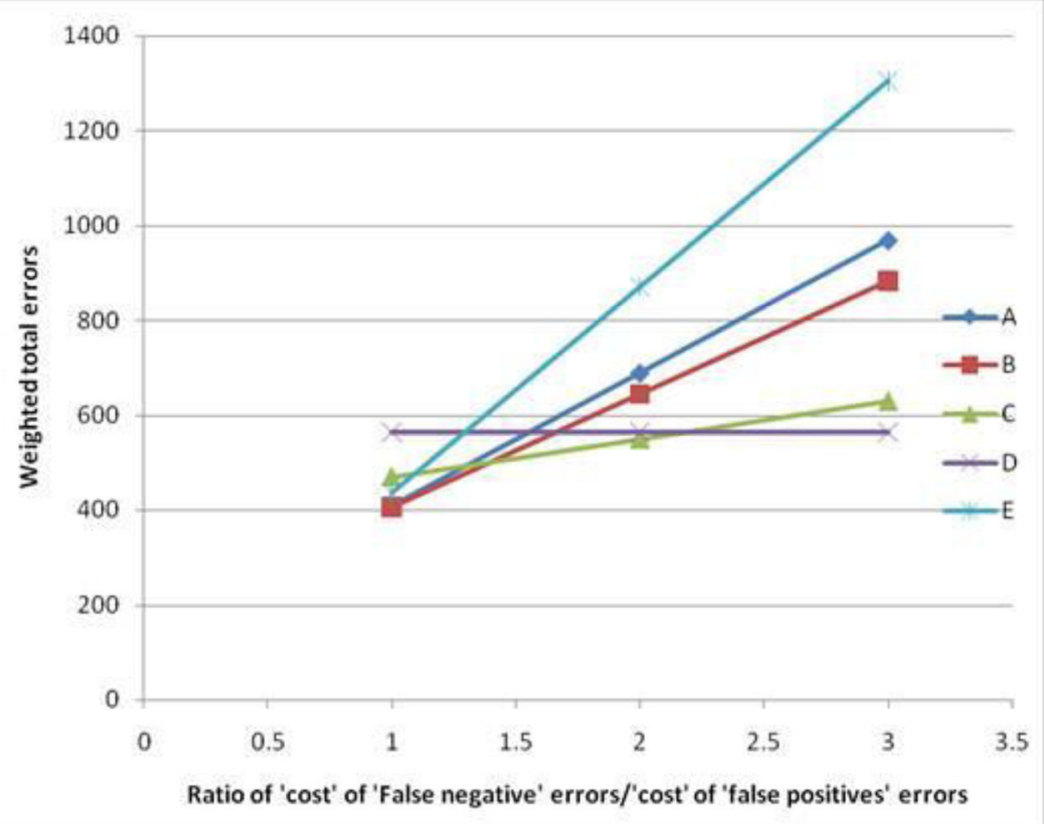

Figure 1.

Total weighted errors associated with screening for any precancerous lesion by the relative 'cost' of 'false negative' errors compared to 'false positive' errors. (A-C: Screening based on risk score with a cut-off targeting $25 \%, 50 \%$ and $75 \%$ of women for A, B and C respectively; D: Universal screening; E: No screening). Note that 'cost' is used as a generic term, not just limited to monetary value, while 'false positive' and 'false negative' respectively refer to screening a patient with no lesion and not screening a patient with lesions. 


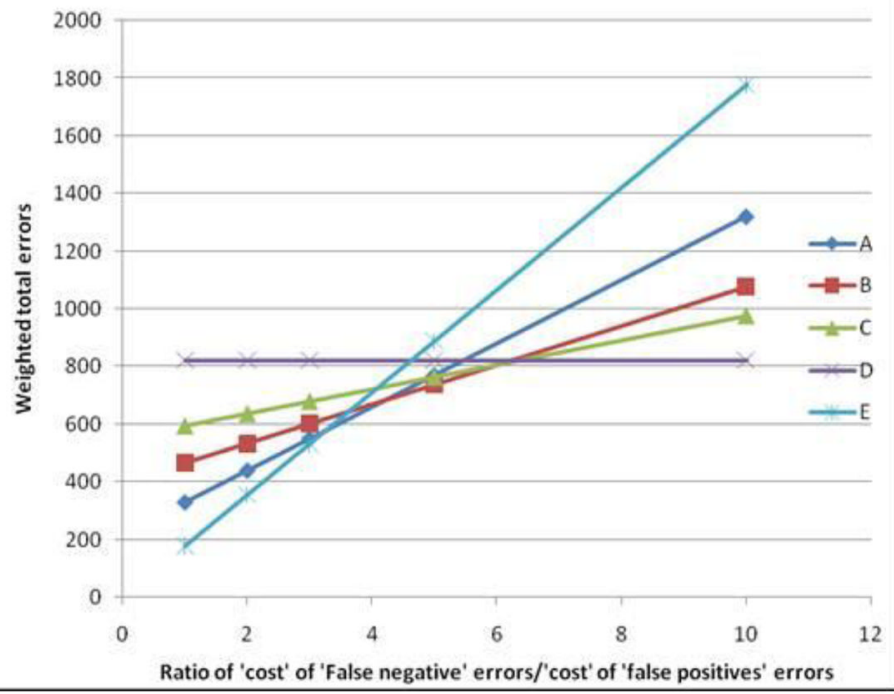

Figure 2.

Total weighted errors associated with screening for ASC-H/HSIL by the relative 'cost' of 'false negative' errors compared to 'false positive' errors. (A-C: Screening based on risk score with a cut-off targeting $25 \%, 50 \%$ and $75 \%$ of women for A, B and C respectively; D: Universal screening; E: No screening). Note that 'cost' is used as a generic term, not just limited to monetary value, while 'false positive' and 'false negative' respectively refer to screening a patient with no severe lesion and not screening a patient with severe lesions. 


\section{Table 1}

Socio-demographic and clinical characteristics in 282 women initiating HAART in Cameroon

\begin{tabular}{|c|c|c|c|}
\hline Characteristic & Level & $\mathbf{N}$ & $\%$ \\
\hline \multirow[t]{7}{*}{ Marital status } & Never married & 60 & 21.3 \\
\hline & Married monogamous & 56 & 19.9 \\
\hline & Married polygamous & 13 & 4.6 \\
\hline & Living with a partner & 30 & 10.6 \\
\hline & Separated & 31 & 11.0 \\
\hline & Divorce & 16 & 5.7 \\
\hline & Widow & 76 & 26.9 \\
\hline \multirow[t]{4}{*}{ Education } & None & 14 & 5.0 \\
\hline & Primary & 135 & 47.9 \\
\hline & Secondary & 121 & 42.9 \\
\hline & Tertiary & 12 & 4.3 \\
\hline \multirow[t]{2}{*}{ Residence } & Urban & 207 & 73.4 \\
\hline & Rural & 75 & 26.6 \\
\hline \multirow[t]{2}{*}{ Previous use of hormonal pills } & Yes & 67 & 23.8 \\
\hline & No & 215 & 76.2 \\
\hline \multirow[t]{4}{*}{ Exposure to tobacco smoke } & No & 141 & 50.0 \\
\hline & Active & 7 & 2.5 \\
\hline & Passive & 133 & 47.2 \\
\hline & Missing & 1 & 0.3 \\
\hline \multirow[t]{4}{*}{ WHO HIV clinical stage } & I & 8 & 2.8 \\
\hline & II & 46 & 16.3 \\
\hline & III & 168 & 59.6 \\
\hline & IV & 60 & 21.3 \\
\hline \multirow[t]{3}{*}{ Previous Pap smear } & No & 269 & 95.4 \\
\hline & Yes & 6 & 2.1 \\
\hline & Don't know/missing & 7 & 2.5 \\
\hline \multirow[t]{7}{*}{ Lifetime sex partners } & 1 & 9 & 3.2 \\
\hline & 2 & 29 & 10.3 \\
\hline & 3 & 56 & 19.9 \\
\hline & 4 & 34 & 12.1 \\
\hline & 5 & 35 & 12.4 \\
\hline & $6+$ & 71 & 25.2 \\
\hline & Missing & 48 & 17.0 \\
\hline \multirow[t]{2}{*}{ Sex partners since HIV diagnosis } & 0 & 127 & 45.0 \\
\hline & 1 & 136 & 48.2 \\
\hline
\end{tabular}




\begin{tabular}{llrr}
\hline Characteristic & Level & N & \% \\
\hline \multirow{2}{*}{ Lifetime condom use } & $2+$ & 19 & 6.8 \\
& Never & 102 & 36.2 \\
& Less than 50\% of the time & 138 & 48.9 \\
& More than 50\% of the time & 39 & 13.8 \\
& Always & 3 & 1.1 \\
\hline \multirow{2}{*}{ Condom use since HIV diagnosis } & Never & 29 & 10.3 \\
& Less than 50\% of the time & 37 & 13.1 \\
& More than 50\% of the time & 19 & 6.7 \\
& Always & 69 & 24.5 \\
& No new partner/missing & 128 & 45.4 \\
\hline \multirow{2}{*}{ History of genital warts } & No & 140 & 49.6 \\
& Yes & 20 & 7.1 \\
& Don't Know/missing & 122 & 43.3 \\
\hline
\end{tabular}




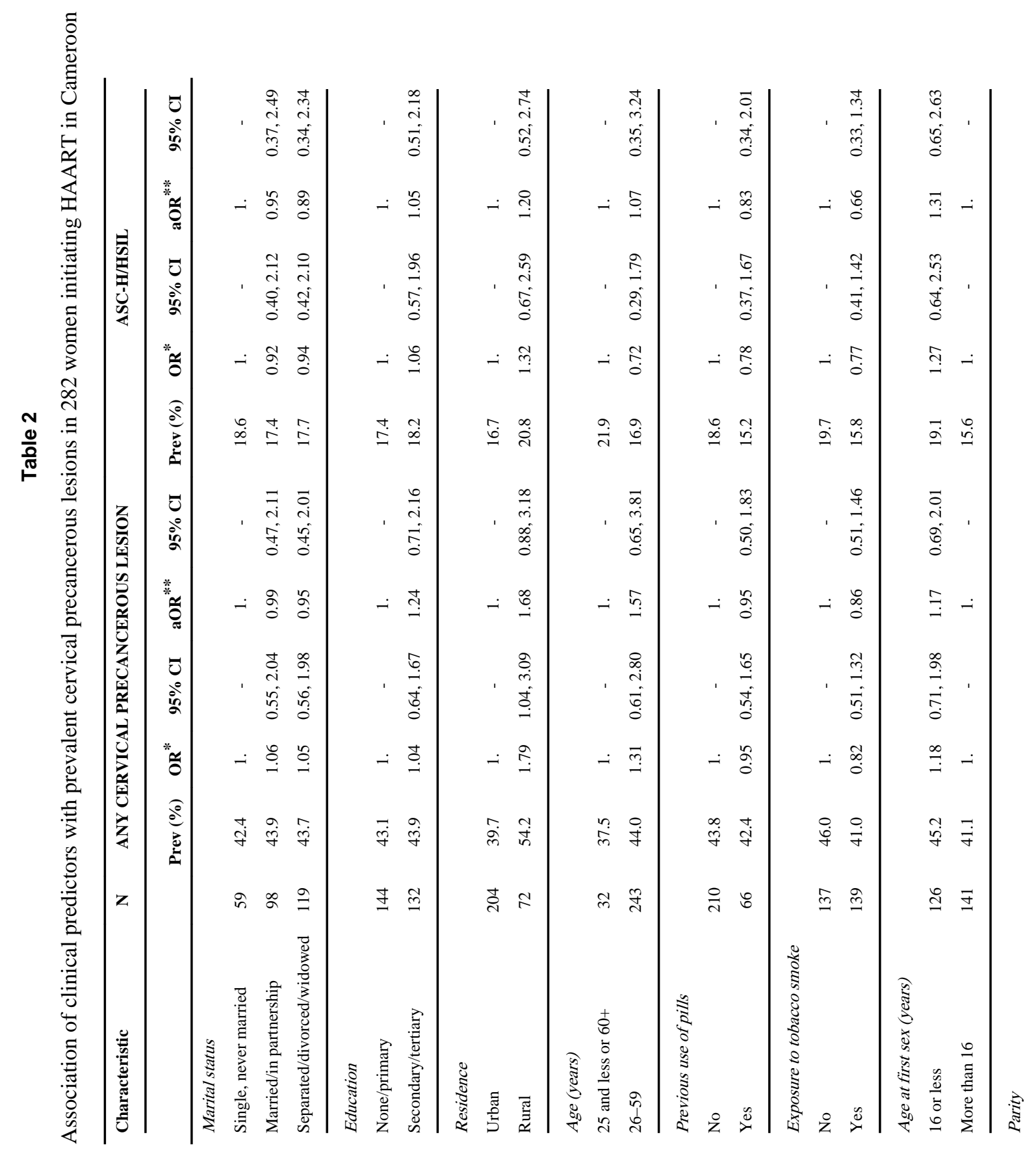


Atashili et al.

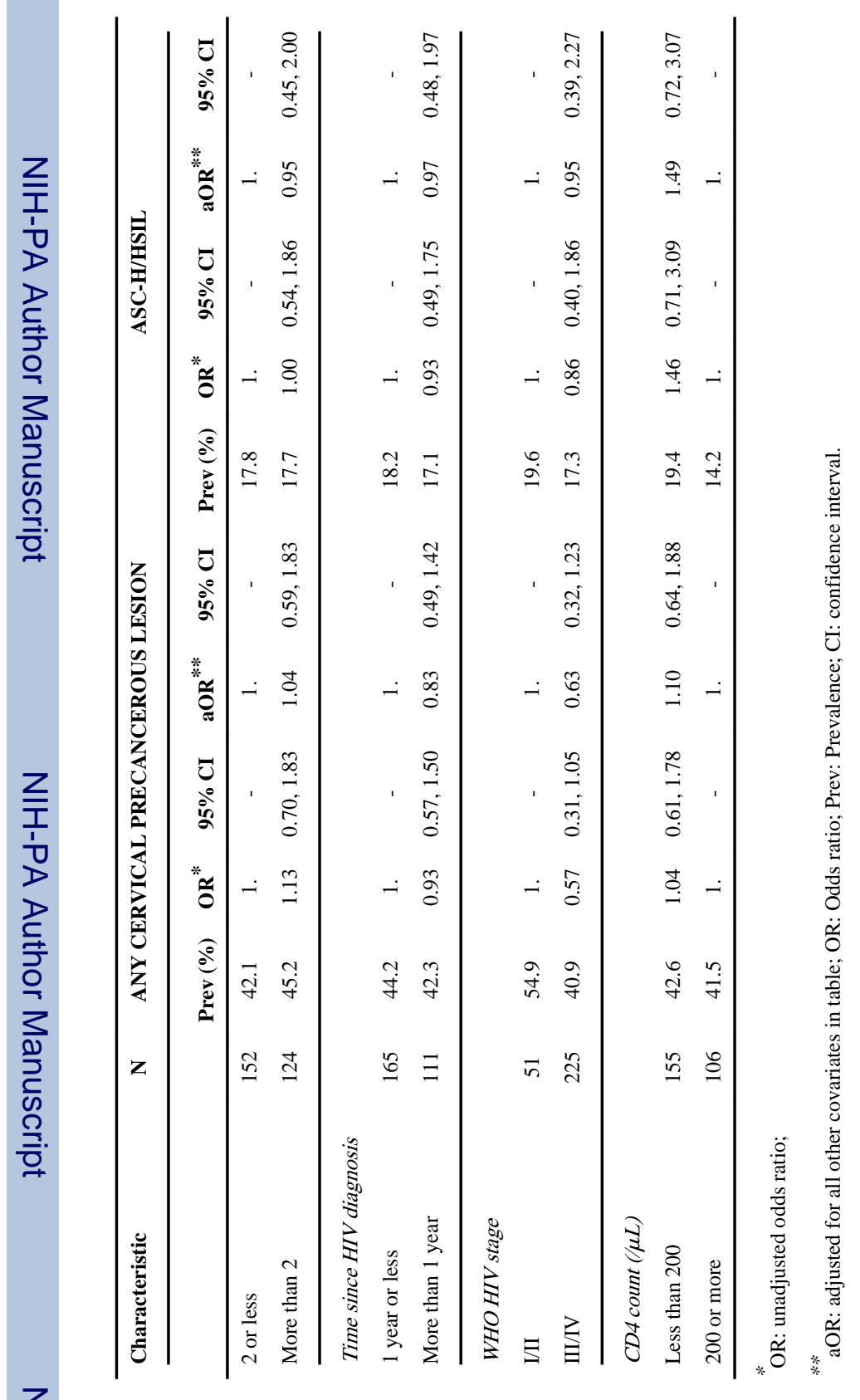

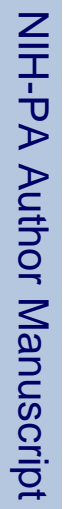




\section{Table 3}

Definition of clinical risk scores developed and assessed for predicting the prevalence of cervical precancerous lesions in women initiating HAART in Cameroon

\begin{tabular}{|l|c|}
\hline Characteristic & Score \\
\hline Full model for predicting prevalent SIL & \\
\hline HIV diagnosed within 1 year & 2 \\
Age at first sex less than or equal 16 years & 2 \\
Not exposed to tobacco & 2 \\
Secondary/tertiary education & 2 \\
Rural residence & 5 \\
WHO HIV Stage I/II & 5 \\
Age 26-59 years & 5 \\
\hline TOTAL & $\mathbf{2 3}$ \\
\hline Full model for predicting prevalent ASC-H/HSIL & \\
\hline Neither separated/widowed/divorced & 1 \\
Aged 26-59 & 1 \\
Rural residence & 2 \\
No previous use of pills & 3 \\
Age at first sex less than or equal 16 years & 4 \\
Not-exposed to tobacco & $\mathbf{1 7}$ \\
CD4 count less than 200 & \\
\hline TOTAL & \\
\hline
\end{tabular}




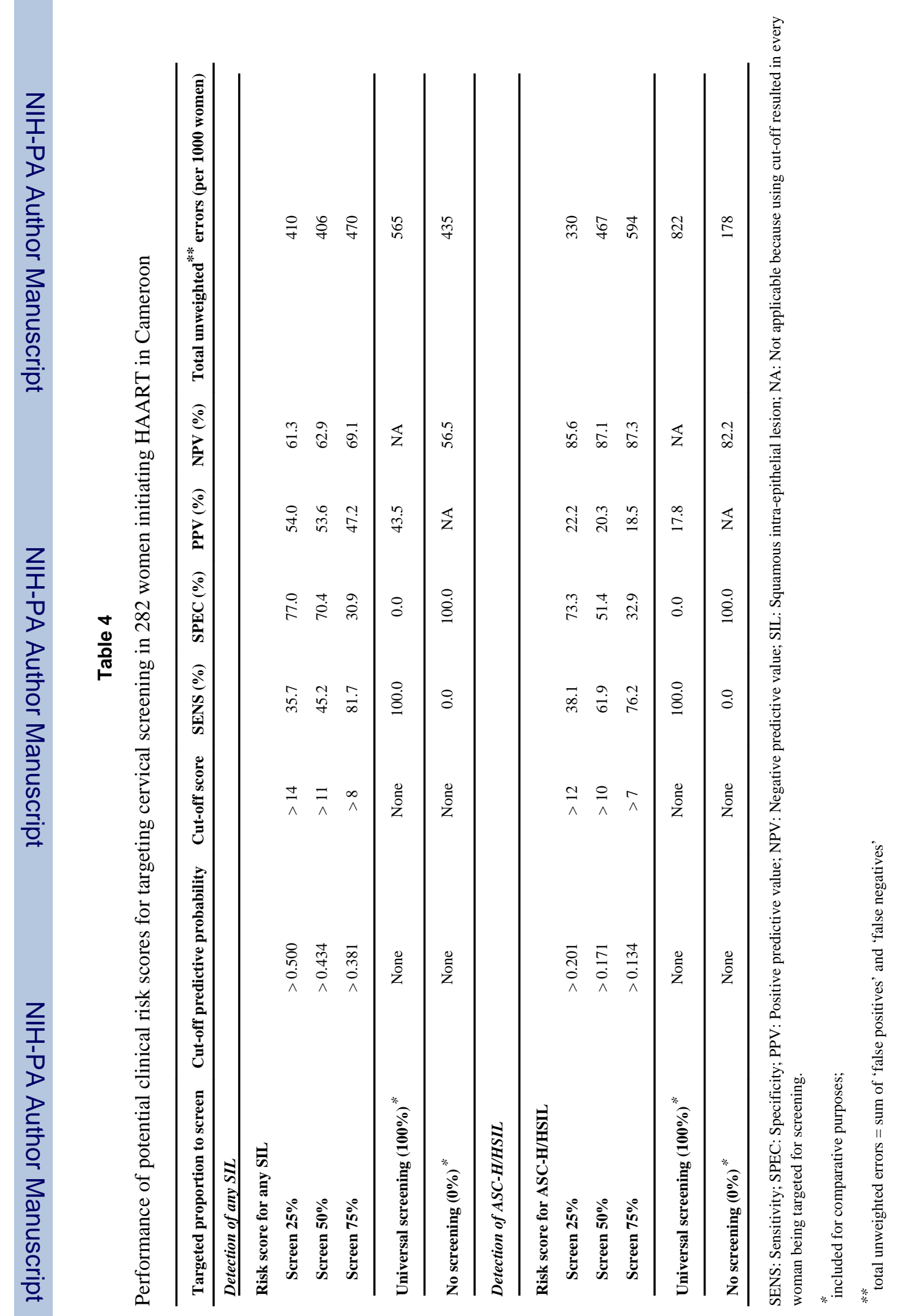

